\title{
Función reflexiva y neurocognición en psicosis: un estudio comparativo
}

\author{
Alicia Ruiz-Toca \\ Hospital Sant Joan de Deu, Lleida (España)
}

Los últimos avances en cognición social en personas con esquizofrenia ponen el énfasis en considerar la función reflexiva como un posible marcador de enfermedad, sin embargo son pocos los estudios que han considerado la influencia de variables neurocognitivas sobre el procesamiento emocional. El objetivo de este trabajo es analizar la velocidad de procesamiento, atención y tiempo de reacción y su relación con la función reflexiva en personas con esquizofrenia y grupo control.

Método: Se reclutaron un total de 190 sujetos; 90 pacientes con diagnostico DSM-V de esquizofrenia y 100 controles sanos. Se recogieron datos clínicos y se midieron las siguientes variables: velocidad de procesamiento, atención, tiempo de reacción, función reflexiva y funcionalidad. Resultados: Los pacientes mostraron un rendimiento más bajo tanto en pruebas cognitivas como en función reflexiva, salvo en hipermentalización ( $\mathrm{p}>$.05). Los pacientes con peor rendimiento en velocidad de procesamiento, presentaron más errores en FR. La funcionalidad se relacionó con FR, velocidad de procesamiento y atención. Discusión: Las dificultades en función reflexiva propias de los pacientes con esquizofrenia podrían estar implicadas en el deterioro funcional global a través de su relación con procesos cognitivos igualmente afectados en estos pacientes.

Palabras clave: Función reflexiva, cognición social, cognición, velocidad de procesamiento, esquizofrenia.

Reflexive function and neurocognition in psychosis. Previous studies in social cognition in people with schizophrenia have considered reflexive function as a possible marker of disease, however there are few studies that have considered the influence of neurocognitive variables on emotional processing. Aim: to analyze the processing speed, attention and reaction time and its relationship with the reflexive function in people with schizophrenia and control group. Method: A total of 190 subjects were recruited; 90 outpatients with DSM-V diagnosis of schizophrenia and 100 healthy controls. Clinical data were collected and following variables were measured: processing speed, attention, reaction time, reflexive function and global functioning. Results: Patients showed a lower performance in both cognitive and reflexive tests, except in hypermentalization ( $p>.05$ ). Patients with worse processing speed performance had more errors in RF. Global functioning was related to FR, processing speed and attention. Discussion: Difficulties in reflective function of patients with schizophrenia could be involved in global functioning deterioration through their relationship with cognitive processes affected in these patients.

Keywords: Reflexive function, social cognition, cognition, processing speed, schizophrenia.

Correspondencia: Alicia Ruíz-Toca. Facultativo Especialista de Área, Psicóloga Clínica. Hospital Sant Joan de Deu. Avda. de la Canadiense 28. C.P.: 25001. Lleida (España). E-mail: aliciaruiztoca@gmail.com 
En las últimas decádas se ha desarrollado una amplia línea de investigaciones acerca de las dificultades en cognición social de las personas con esquizofrenia (Irani, Seligman, Kamath, Kohler, y Gur, 2012; Penn, Sanna, y Roberts, 2008; Savla, Vella, Armstrong, Penn, y Twamle, 2013). Dentro del marco de la cognición social, se define la función reflexiva (FR), así como sus conceptos relacionados (mentalización o Teoría de la Mente-TdM-), como la capacidad que permite interpretar las intenciones, los deseos, sentimientos y creencias, propios y ajenas y por ende, predecir y explicar la propia conducta y de los otros (Fonagy, Gergely, Jurist, y Target, 2002; Fonagy, Steele, Moran, Steele, y Higgit, 1991). Algunos autores consideran los déficits en función reflexiva como marcadores de rasgos de enfermedad (Brüne, 2005) y sitúan la esquizofrenia como una alteración en la capacidad de representar adecuadamente los estados mentales propios y ajenos (Frith, 1992). Por este motivo, algunos trabajos han centrado su interés en desarrollar estrategias de evaluación con mayor validez ecológica y discriminante de los procesos superiores de la cognición social como la FR, a través de instrumentos audiovisuales dinámicos que se aproximen a los contextos y escenas cotidianas reales (Montag, 2011).

Los últimos estudios en cognición social han considerado las dificultades en cognición tradicionales de las personas con esquizofrenia, como la velocidad de procesamiento o la atención, y su impacto sobre el funcionamiento global en resolucion de problemas de la vida diaria, rendimiento laboral o habilidades sociales (Andersen, et al., 2013; Fioravanti, Bianchi, y Cinti, 2012; Gold, y Thaker, 2002; Harvey, Keefe, Patterson, Heaton, y Bowie, 2009; Revheim et al., 2006). Sin embargo, aun son pocos los estudios que ponen en relación el influjo de la neurocognición como variable mediadora en el procesamiento emocional y funcionamiento global (Lahera, Ruiz, Brañas, Vicens, y Orozco, 2017; Tsotsi, Bozikas, y Kosmidis, 2015).

El objetivo del presente trabajo es analizar el rendimiento en variables neurocognitivas como la velocidad de procesamiento, atención y tiempo de reacción y su relación con el procesamiento emocional medido a través de una prueba de función reflexiva en personas con esquizofrenia y personas sin enfermedad.

\section{MÉTODO}

\section{Participantes}

Se reclutaron una muestra total de 190 sujetos con edades comprendidas entre 18 y 65 años. La muestra de pacientes estaba compuesta por 90 pacientes ambulatorios con diagnóstico de esquizofrenia de acuerdo al Diagnostic Statistical Manual V Edition (DSM-V), (American Psychiatric Association, 2013). Todos los pacientes estaban clínicamente estables durante los últimos tres meses. Fueron excluidos los pacientes que cumplían criterios para otros diagnósticos, aquellos con datos de impregnación 
farmacológica moderada-severa según la Escala de Simpson-Angus (por su potencial impacto en el tiempo de reacción), retraso mental, enfermedad neurológica, historia de traumatismo craneoencefálico, enfermedad médica grave, analfabetismo funcional, abuso de sustancias psicoactivas exceptuando el tabaco o negativa a participar. El comité de ética del Hospital Universitario Príncipe de Asturias aprobó el protocolo de evaluación y todos los participantes firmaron el consentimiento informado.

El grupo de sujetos control estaba compuesto por 100 sujetos sin patología neurológica o psiquiátrica ni seguimiento en salud mental en los últimos 12 meses y presentaba las mismas condiciones sociodemográficas que el grupo experimental.

Se recogieron datos sociodemográficos y clínicos (Positive and Negative Syndrome Scale -PANSS-), y se midieron las variables: velocidad de procesamiento (test del trazo -TMT-, codificación de símbolos -BACS-, fluidez verbal -animales-), atención sostenida y tiempo de reacción (Continuous Performance Test-CPT-), función reflexiva (Movie Assesment Social Cognition -MASC-) y funcionalidad (Escala de Funcionamiento Personal y Social -PSP-).

\section{Instrumentos}

La evaluación clínica se llevó a cabo con la Escala para el Síndrome Positivo y Negativo de la Esquizofrenia (PANSS) (Kay, Opler, y Lindenmayer, 1989); versión española (Peralta-Martín, y Cuesta-Zorita, 1994). Para detectar la presencia y gravedad de la sintomatología parkinsoniana se aplicó la Escala de Simpson Angus (SAS) (Simpson y Angus, 1970); versión española de Calvo-Gómez, Sánchez-Pedraza, Jaramillo-González, y Tarcisio-Mantilla, (2006). La valoración de la funcionalidad global se realizó mediante la Escala de Personal and Social Performance (PSP) (Morosini, Magliano, Brambilla, Ugolini y Pioli, 2000) validación española de García-Portilla, (2011).

Para la evaluación cognitivas se utilizaron los siguientes instrumentos: La velocidad de procesamiento se evaluó valorando el rendimiento en tres pruebas: a) Subtest de codificación de símbolos (Brief Assessment of Cognition in Schizophrenia; BACS) (Keefe, 1999), b) Test del Trazo-A (Trail Making Test; TMT-A), (Reitan, y Wolfson, 1993); y c) Prueba de fluencia verbal (categoría “Animales"), (Benton, y Hamscher, 1978). La atención sostenida y el tiempo de reacción se evaluaron mediante el Test de Ejecución Continua (Continuous Performance Test, CPT), (Cornblatt, Risch, Faris, Friedman, y Erlenmeyer-Kimling, 1988).

Para la evaluación de la FR se utilizó la Movie for the assessment of social

cognition (MASC) (Dziobek et al., 2006), Versión castellana MASC de Lahera et al. (2014). Instrumento audiovisual de 45 minutos que evalúa la cognición social a partir de una película en la que se muestra la interacción entre diferentes personajes. La imagen se detiene en 46 ocasiones, preguntando al sujeto acerca de las 
emociones, pensamientos e intenciones que atribuyen a los protagonistas en cada escena. Da como resultado cuatro puntuaciones en cognición social: atribución correcta en la capacidad de mentalizar (aciertos), exceso de atribución en la capacidad de mentalizar (hipermentalización o hiperTdM), déficit de atribución en la capacidad de mentalizar (hipomentalización o hipoTdM) y ausencia de atribución en la capacidad de mentalizar (no mentalización o no TdM). La MASC ha demostrado un alto poder discriminativo en detectar déficits en TdM comparado con otras pruebas de cognición social que utilizan situaciones ejemplo o imágenes estáticas, una elevada fiabilidad interevaluadores y alta consistencia interna (Montag et al., 2011).

\section{Procedimiento}

Los sujetos fueron evaluados de manera individual por un psiquiatra o psicólogo clínico durante aproximadamente 50 minutos, en el centro ambulatorio de referencia del paciente, en el caso de los pacientes y en lugares comunitarios en el caso de los controles.

\section{Análisis de datos}

Se trata de un estudio observacional transversal de tipo caso-control. Se utilizó la media y desviación estándar para la descripción de datos continuos, y los porcentajes para datos categóricos. Con respecto a las variables cuantitativas, se determinó su ajuste a la distribución normal mediante la prueba de Kolmogorov-Smirnov. Para comparar la diferencia de medias se utilizaron el análisis de la t de student y la U de Mann-Whitney, según el comportamiento de las variables estudiadas. La asociación entre variables se analizó a través de la correlación parcial de Pearson, controlando posibles variables confusoras (como edad o equivalentes de clorpromacina). El análisis estadístico de los datos se realizó mediante el paquete estadístico SPSS ${ }^{39}$, versión 22.0. El nivel de significación fijado para todas las pruebas de contraste de hipótesis del 0.05.

\section{RESULTADOS}

\section{Variables Cognitivas}

Los pacientes participantes en el estudio tenían una edad media de 44.14 años $(D E: 9.7)$ en el grupo experimental y de 41.5 años $(D E: 12.1)$ en el grupo control. En el grupo experimental el $24.4 \%$ eran mujeres (75.6\% hombres) frente al $37 \%$ de mujeres (63\% hombres) en el grupo control. El $75.6 \%$ estaba soltero, el $17.8 \%$ estaba casado y el $6.7 \%$ estaba separado o viudo, en el grupo experimental, mientras que en el grupo control $54 \%$ estaba soltero, el $38 \%$ estaba casado y el $8 \%$ estaba separado o viudo. Respecto al nivel de estudios, en el grupo experimental el $41.1 \%$ tenía estudios básicos, el $44.4 \%$ secundarios y el $12.2 \%$ universitarios, mientras que en el grupo control $28 \%$ tenía estudios 
básicos, el 53\% secundarios y el 19\% universitarios. En el ámbito laboral el 17.8\% de los pacientes tenía un empleo, el $24.4 \%$ estaba desempleado y el 56.7 era pensionista, mientras que dentro del grupo control el $80 \%$ tenía un empleo, el $12 \%$ estaba desempleado y el $6 \%$ era pensionista. La caracterización clínica de la muestra se expone en la tabla 1.

Se encontraron algunas relaciones estadísticamente significativas entre las variables sociodemográficas y las variables estudiadas. La edad se relacionaba con velocidad de procesamiento (TMTa: $P=0.575, p=0.000$ y BACS: $P=-0.442, p=0.000$ ), con atención $(P=-0.405, p=0.000)$ y tiempo de reacción $(P=0.286, p=0.007)$, con el total de aciertos en función reflexiva (MASC: $\mathrm{P}=-0,280, \mathrm{p}=0,008)$ y con funcionalidad global $(P=-0.435 ; p=0.000)$. Los años de evolución de la enfermedad se relacionaron con velocidad de procesamiento (TMTa: $P=0.339, p=0.001$ y BACS: $P=-0.234, p=0.026$ ), atención sostenida $(P=-0.279, p=0.008)$, con funcionalidad $(P=-0.276, p=0.008)$. No se encontró relación entre tiempo de evolución y rendimiento en función reflexiva. El número de ingresos no mostró relación con ninguna variable estudiada.

Tabla 1. Análisis descriptivo de las variables sociodemográficas y clínicas de la muestra: media, SD y porcentajes

\begin{tabular}{lcc}
\hline & Pacientes $(\mathrm{n}=90)$ & G. Control $(\mathrm{n}=100)$ \\
\hline Edad & $44,14(9,7)$ & $41,5(12,1)$ \\
\hline Mujeres & $24,4 \%$ & $37 \%$ \\
\hline Estudios Superiores & $12,2 \%$ & $19 \%$ \\
\hline Activo (Actividad laboral) & $52 \%$ & $18 \%$ \\
\hline Entrenamiento en HHSS y Cognición & $4,3 \%$ & $1,5 \%$ \\
\hline Antecedentes esquizofrenia familiar & $22,2 \%$ & $10 \%$ \\
\hline Diagnóstico esquizofrenia paranoide & $82,2 \%$ & \\
\hline Años de evolución & $16.9(8.7)$ & \\
\hline Número de ingresos & $2,0(2,5)$ & \\
\hline PANSS Positiva & $9.8(3.2)$ & \\
\hline PANSS Negativa & $10.1(3.1)$ & \\
\hline PANSS General & $20,4(4,5)$ & \\
\hline PANSS Total & $40.4(9.4)$ & \\
\hline ANGUS & $0,7(1,2)$ & \\
\hline PSP & $53,6(15,4)$ & \\
\hline
\end{tabular}

En general, los pacientes mostraron un rendimiento cognitivo en velocidad de procesamiento (TMTa, BACS, Animales), atención sostenida, tiempo de reacción y en función reflexiva (MASC) significativamente más pobre con respecto al grupo control exceptuando la subescala de errores en hipermentalización donde no se encontraron diferencias entre los grupos (ver Tabla 2). En el perfil de errores existen diferencias entre grupo de pacientes y grupo control. El grupo de pacientes muestra una tendencia de errores marcada por la hipomentalización, mientras que en el grupo control, la tendencia de errores predominante es de hipermentalización. 
Tabla 2. Media y DE de puntuaciones en las pruebas cognitivas, aciertos y errores en la MASC. Pacientes $(n=90)$, controles $(n=100)$

\begin{tabular}{llll}
\hline & Grupo Pacientes & Grupo Control & $\mathrm{p}<.05$ \\
\hline TMTa & $42,5(22,6)$ & $25,90(11,48)$ & .000 \\
\hline BACS & $34,86(13,8)$ & $51,08(12,11)$ & .000 \\
\hline Animales & $18,4(6,3)$ & $24,3(6,6)$ & .000 \\
\hline Atención Sostenida & $2.8(1.1)$ & $3,7(0,6)$ & .000 \\
\hline Tiempo de Reacción & $555,6(107,5)$ & $474,9(66,9)$ & .000 \\
\hline MASC Total Aciertos & $20,9(7,1)$ & $29,9(5,3)$ & .000 \\
\hline MASC Total Errores & $23,75(6,98)$ & $14,74(5,59)$ & .000 \\
\hline Errores Hiper TdM & $7,18(3,48)$ & $7,20(3,10)$ & .908 \\
\cline { 2 - 4 }$\%$ Hiper TdM & $31,47(14,95)$ & $50,17(14,60)$ & .000 \\
\hline Errores Hipo TdM & $10,58(4,43)$ & $5,14(2,69)$ & .000 \\
\cline { 2 - 4 }$\%$ Hipo TdM & $44,70(13,46)$ & $35,31(13,46)$ & .000 \\
\hline Errores No TdM & $5.98(3.59)$ & $2,39(2,04)$ & .000 \\
\cline { 2 - 4 }$\%$ No TdM & $23,82(11,43)$ & $14,52(10,09)$ & .000 \\
\hline MASC Tiempo total & $611,2(212,2)$ & $446,9(124,4)$ & .000 \\
\hline Nota. *nivel de significación 0.05 & & &
\end{tabular}

\section{Función reflexiva, variables cognitivas, y sintomatología}

Se encontró una relación estadísticamente significativa entre la subescala PANSS negativa y la velocidad de procesamiento medida a través de la prueba Animales $(P=-0.252 ; p=0.016)$ y tiempo de reacción $(P=0.249 ; p=0.019)$. Se encontró una tendencia de relación entre la subescala PANSS General y la velocidad de procesamiento con la prueba Animales $(P=-0.200 ; p=0.059)$. No se encontró una correlación estadísticamente significativa entre la puntuación en la subescala PANSS positiva y las variables cognitivas, ni entre el rendimiento en función reflexiva, el tiempo de ejecución de la prueba de función reflexiva (MASC) y las subescalas PANSS positiva o PANSS negativa.

\section{Función reflexiva, variables cognitivas y funcionalidad global}

Se encontraron relaciones significativas en todas las variables cognitivas analizadas y la función reflexiva (ver Tabla 3). El total de aciertos en la prueba MASC se relacionó con el tiempo de ejecución de la prueba, velocidad de procesamiento y atención sostenida, con una tendencia de relación en tiempo de reacción. El total de errores en MASC se relacionó con el tiempo de ejecución en la prueba, velocidad de procesamiento, atención sostenida y una tendencia de relación en tiempo de reacción. Los errores por hipermentalización se relacionan con velocidad de procesamiento (TMTa y BACS) y con atención sostenida, mientras que los errores por hipomentalización se relacionan únicamente con velocidad de procesamiento (BACS) y con el tiempo de ejecución en la prueba. La no mentalización se relacionó con el tiempo de ejecución en la prueba, velocidad de procesamiento y con atención sostenida. De igual manera, el tiempo de ejecución en la prueba MASC, se relacionó con la velocidad de procesamiento (TMTa: $P=.234 ; p=.028$, Animales: $P=-.347 ; p=.001$ y BACS: $P=-.288 ; p=.006$ ) у con tiempo de reacción $(P=0.316 ; p=.003)$. No se encontró relación entre el tiempo de ejecución de la MASC con atención sostenida ni con funcionalidad. La funcionalidad se 
relaciona con aciertos, y el total de errores, así como con errores por hipomentalización. En las variables cognitivas las funcionalidad se relaciona con velocidad de procesamiento (TMTa: $P=-0.269 ; p=0.010$, BACS: $P=0.348 ; p=0.001)$ y con atención $(P=0.223$; $p=0.036)$.

Tabla 3. Correlaciones entre variables cognitivas, funcionalidad, tipos de errores y tipos de aciertos en la MASC. Pacientes $(n=88)$ y controles $(n=100)$

\begin{tabular}{|c|c|c|c|c|c|}
\hline & Total Aciertos & Total Errores & Hiper TdM & Hipo TdM & No TdM \\
\hline \multirow[t]{2}{*}{ TMTa } & $\mathrm{P}=-0.401$ & $\mathrm{P}=0.410$ & $\mathrm{P}=0.281$ & $\mathrm{P}=0.196$ & $\mathrm{P}=0.330$ \\
\hline & $\mathrm{p}=0.000^{*} *$ & $\mathrm{p}=0.000 * *$ & $\mathrm{p}=0.008^{*} *$ & $\mathrm{p}=0.066$ & $\mathrm{p}=0.002 * *$ \\
\hline \multirow[t]{2}{*}{ Animales } & $\mathrm{P}=0.397$ & $\mathrm{P}=-0.376$ & $\mathrm{P}=-0.181$ & $\mathrm{P}=-0.146$ & $\mathrm{P}=-0.421$ \\
\hline & $\mathrm{p}=0.000^{* *} *$ & $\mathrm{p}=0.000 * *$ & $\mathrm{p}=0.091$ & $\mathrm{p}=0.174$ & $\mathrm{p}=0.000^{* * *}$ \\
\hline \multirow[t]{2}{*}{ BACS } & $\mathrm{P}=0.549$ & $\mathrm{P}=-0.549$ & $\mathrm{P}=-0.299$ & $\mathrm{P}=-0.374$ & $\mathrm{P}=-0.375$ \\
\hline & $\mathrm{p}=0.000^{* *}$ & $\mathrm{p}=0.000 * *$ & $\mathrm{p}=0.005^{* *}$ & $\mathrm{p}=0.000 * *$ & $\mathrm{p}=0.000^{* *}$ \\
\hline \multirow[t]{2}{*}{$\overline{\mathrm{AS}}$} & $\mathrm{P}=0.373$ & $\mathrm{P}=-0.384$ & $\mathrm{P}=-0.221$ & $\mathrm{P}=-0.173$ & $\mathrm{P}=-0.370$ \\
\hline & $\mathrm{p}=0.000^{* *}$ & $\mathrm{p}=0.000^{* *}$ & $\mathrm{p}=0.040^{*}$ & $\mathrm{p}=0.110$ & $\mathrm{p}=0.000^{* * *}$ \\
\hline \multirow[t]{2}{*}{ TR } & $\mathrm{P}=-0.203$ & $\mathrm{P}=0.197$ & $\mathrm{P}=0.082$ & $\mathrm{P}=0.162$ & $\mathrm{P}=0.125$ \\
\hline & $\mathrm{p}=0.060$ & $\mathrm{p}=0.068$ & $\mathrm{p}=0.448$ & $\mathrm{p}=0.134$ & $\mathrm{p}=0.251$ \\
\hline \multirow[t]{2}{*}{ Funcionalidad } & $\mathrm{P}=0.231$ & $\mathrm{P}=-0.218$ & $\mathrm{P}=0.068$ & $\mathrm{P}=-0.298$ & $\mathrm{P}=-0.136$ \\
\hline & $\mathrm{p}=0.001 * *$ & $\mathrm{p}=0.041 *$ & $\mathrm{p}=0.528$ & $\mathrm{p}=0.005^{* *}$ & $\mathrm{p}=0.206$ \\
\hline \multirow[t]{2}{*}{ Tiempo total } & $\mathrm{P}=-0.359$ & $\mathrm{P}=0.378$ & $\mathrm{P}=0.154$ & $\mathrm{P}=0.279$ & $\mathrm{P}=0.281$ \\
\hline & $\mathrm{p}=0.001^{*} *$ & $\mathrm{p}=0.000 * *$ & $\mathrm{p}=0.151$ & $\mathrm{p}=0.008 * *$ & $\mathrm{p}=0.008 * *$ \\
\hline
\end{tabular}

Nota. *nivel de significación 0.05

\section{DISCUSIÓN/CONCLUSIONES}

El presente trabajo trata de analizar la relación entre la función reflexiva y aquellas variables neurocognitivas que se han visto demostradamente afectadas en los pacientes con esquizofrenia. La aportación fundamental del estudio es mostrar una relación significativa entre el tiempo de procesamiento y la ejecución en el reconocimiento de emociones e intenciones en pacientes con esquizofrenia. En este sentido, como han mostrado algunos estudios recientes (Lahera, Ruiz, Brañas, Vicens, y Orozco, 2017), los pacientes que mostraron peor rendimiento en velocidad de procesamiento, así como un tiempo de ejecución más dilatado en la prueba de cognición social y función reflexiva MASC, presentaron mayor número de errores en función reflexiva. Este hecho es comprensible si se considera que a mayor tiempo de procesamiento necesario para identificar, entender e integrar toda la información disponible, mayores dificultades existirán para producir una respuesta acorde con la situación social. Estudios anteriores, habían examinado la relación entre función reflexiva y variables cognitivas de segundo orden como el aprendizaje verbal, no observando relaciones significativas con la función reflexiva (Montag et al., 2011). Sin embargo, este estudio valora funciones cognitivas de primer orden como la velocidad de procesamiento, una capacidad que se encuentra implicada y es transversal a todas las actividades cognitivas, ya sea con material social o no social.

El análisis pormenorizado de la función reflexiva en el grupo experimental demostró un perfil de errores claramente diferente respecto al perfil de errores en el grupo 
control. El grupo de pacientes mostró una mayor tendencia de error en hipomentalización, mientras que las personas sin enfermedad manifestaron una tendencia de errores predominante en hipermentalización. De esta forma, tal y como se ha observado en anteriores estudios (Horan, Nuechterlein, Wynn, Lee, Castelli y Green, 2009; Montag et al., 2011) los pacientes con esquizofrenia presentan un déficit en función reflexiva con dificultades para detectar e integrar toda la información disponible a nivel verbal, no verbal o contextual para establecer una conclusión congruente con la escena. No obstante y a pesar de los perfiles diferenciales en los errores en mentalización entre pacientes y grupo control, no se observaron diferencias en errores por hipermentalización, tal y como han demostrado otros estudios (Montag et al., 2011). En cuanto a las relaciones entre los tipos de errores en función reflexiva y variables cognitivas en el grupo de pacientes se observó que la velocidad de procesamiento se relacionaba tanto con errores por hipermentalización, como con errores por hipo mentalización. Sin embargo el rendimiento en atención sostenida solo se relacionó con los errores en hipermentalización. Estos datos sugieren que las dificultades para atender a la información disponible a nivel social favorece una interpretación más elaborada de la situación, en un esfuerzo por completar la información no atendida, mientras que un déficit en teoría de la mente no se asociaría tanto a las dificultades para atender en el contexto social, sino para captar el conjunto de los datos de la escena social de una forma lógica, comprensible y coherente (Tsotsi, Bozikas y Kosmidis, 2015). Por último señalar en cuanto a posibles variables mediadoras entre la función reflexiva y la neurocognición, no se observaron relaciones entre los síntomas de enfermedad medidos con la PANSS y la función reflexiva a diferencia de otros estudios realizados sobre esta variable, debido en parte, a la condición de estabilidad clínica necesaria para ser incluidos dentro del estudio. La funcionalidad global, tal y como se ha demostrado en estudios recientes (Lahera, Ruiz, Brañas, Vicens, y Orozco, 2017) mostró, sin embargo, relaciones significativas con velocidad de procesamiento y con la función reflexiva, sobre todo en errores de hipomentalización, aquellos que son más predominantes en el grupo de pacientes, mientras que no se encontró relación con errores por hipermentalización, presentes en ambos grupos. Esto es coherente con la idea de que las dificultades en función reflexiva propia de los pacientes con esquizofrenia podrían estar implicadas en el deterioro funcional global a través de su relación con procesos cognitivos transversales igualmente afectados en estos pacientes.

En el presente trabajo existen una serie de fortalezas y limitaciones. Entre las fortalezas del estudio cabe mencionar: a) el análisis de la relación, hasta el momento escasamente estudiado sobre capacidades cognitivas superiores, como la velocidad de procesamiento y la función reflexiva, entendida como la capacidad para inferir estados emocionales e intenciones en los otros, b) el tamaño representativo de la muestra de pacientes junto con el tamaño representativo del grupo control, c) la utilización de instrumentos de cognición social audiovisuales, ecológicos y dinámicos, más 
representativos de escenas sociales reales donde se encuentra siempre presente el tiempo de la interacción y los cambios cognitivos rápidos entre las personas. En cuanto a las limitaciones cabe señalar: a) no inclusión de algunas variables cognitivas importantes en el estudio de pacientes con esquizofrenia como la memoria y la función ejecutiva, b) analizar de forma aislada la capacidad de lectoescritura de los pacientes para evitar sesgos en las respuestas por interferencia de variables contaminadoras en la prueba MASC, c) considerar el análisis de las variables cognitivas y la función reflexiva de forma correlacional y el papel mediador sobre la función global de cada una de ellas. Futuros estudios deberán desarrollar líneas de trabajo que clarifiquen la dirección de estas relaciones.

\section{REFERENCIAS}

Andersen, R., Fagerlund, B., Rasmussen, H., Ebdrup, B.H., Aggernaes, B., Gade, A., ... Glenthoj, B. (2013). The influence of impaired processing speed on cognition in first-episode. European Psychiatry, 28, 332-339.

American Psychiatric Association. (2013). Diagnostic and statistical manual of mental disorders (5th ed.). Washington: American Psychiatric Association.

Benton, A.L., y Hamscher, K. (1978). Multilingual aphasia examination manual (revised). University of Iowa: AJA Associates.

Brüne, M. (2005). "Theory of mind" in schizophrenia: a review of the literature. Schizophrenia Bulletin, 13(1), 21-42.

Calvo-Gómez, J.M., Sánchez-Pedraza, R., Jaramillo-González, L.E., y Tarcisio-Mantilla, C. (2006). Validating the Simpson-Angus Extrapyramidal Collateral Symptom Evaluation Scale. Rev Salud Pública (Bogota), 8(1), 74-87. Spanish.

Cornblatt, B.A., Risch, N.J., Faris, G., Friedman, D. y Erlenmeyer-Kimling, L. (1988). The Continuous Performance Test, identical pairs version (CPT-IP): I. New findings about sustained attention in normal families. Psychiatry Res., 26, 223-238.

Dziobek, I., Fleck, S., Kalbe, E., Rogers, K., Hassenstab, J., Brand, M.,... Convit, A. (2006). Introducing MASC: a movie for the assessment of social cognition. Journal of autism and developmental disorders, 36(5), 623-636.

Fioravanti, M., Bianchi, V., y Cinti, M.E. (2012). Cognitive deficits in schizophrenia: an updated metanalysis of the scientific evidence. BMC Psychiatry, 20, 12-64.

Fonagy, P., Gergely, G., Jurist, E., y Target, M. (2002). Affect regulation, mentalization, and the development of the self. New York: Other Press.

Fonagy, P., Steele, M., Moran, G., Steele, H., y Higgit, A. (1991). The capacity for understanding mental states: the reflective self in parent and child and its significance for security of attachment. Infant Mental Health Journal, 13, 201-218.

Frith, C.D. (1992). The Cognitive Neuropsychology of Schizophrenia. UK: Lawrence Erlbaum Associates, Hove.

Garcia-Portilla, M.P., Saiz, P.A., Bousoño, M., Bascaran, M.T., Guzmán-Quilo C., y Bobes, J. (2011). Validation of the Spanish Personal and Social Performance scale (PSP) in outpatients with stable and unstable schizophrenia. Rev Psiquiatr Salud Ment. 4, 9-18.

Gold, J.M. y Thaker, G.K. (2002). Current progress in schizophrenia research: cognitive phenotypes of schizophrenia: attention. J Nerv Ment Dis.190, 638-639. 
Harvey, P.D., Keefe, R.S.E., Patterson, T.L., Heaton, R.K., y Bowie, C.R. (2009). Abbreviated neuropsychological assessment in schizophrenia: Prediction of different aspects of outcom. J Clin Exp Neuropsychol, 31, 462-471.

Horan, W.P., Nuechterlein, K.H., Wynn, J.K., Lee J., Castelli, F. y Green, M.F. (2009). Disturbances in the spontaneous attribution of social meaning in schizophrenia. Psychol Med, 39(4), 635-643.

Irani, F., Seligman, S., Kamath, V., Kohler, C. y Gur, R.C. (2012). A Meta-Analysis of Emotion Perception and Functional Outcomes in Schizophrenia. Schizophr Res, 137, 203-211.

Kay, S.R., Opler, L.A., y Lindenmayer, J.P. (1989). The Positive and Negative Syndrome Scale (PANSS): rationale and standardisation. Br J Psychiatry Suppl, 7, 59-67.

Keefe, R.S.E. (1999). Brief Assesment of cognition in Schizophrenia (BACS) Manual - A: Version 2.1. Duke University Medical Centre: Durham.

Lahera, G., Ruiz, A., Brañas, A., Vicens, M., y Orozco, A. (2017). Reaction time, processing speed and sustained attention in schizophrenia: impact on social functioning. Rev Psiquiatr Salud Ment. doi: 10.1016/j.rpsm.2017.04.001

Lahera, G., Boada, L., Pousa, E., Mirapeix, I., Morón-Nozaleda, G., Marinas, L.,... Parellada, M. (2014). Movie for the Assessment of Social Cognition (MASC): Spanish Validation. Journal of autism and developmental disorders, 44(8), 1886-1896.

Montag., C., Dziobek, I., Richter, I.S., Neuhaus, K., Lehmann, A., Sylla, R.,... Gallinat, J. (2011). Different aspects of theory of mind in paranoid schizophrenia: evidence from a videobased assessment. Psychiatry Research, 186(2-3), 203-209.

Morosini, P.L., Magliano, L., Brambilla, L., Ugolini, S., y Pioli, R. (2000). Development, reliability and acceptability of a new version of the DSM-IV Social and Occupational Functioning Assessment Scale (SOFAS) to assess routine social functioning. Acta Psychiatr Scand. 101, 323-329.

Penn, D.L., Roberts, D.L., Combs, D., y Sterne, A. (2007). The development of the Social Cognition and Interaction Training Program for schizophrenia spectrum disorders. Psychiatric Services, 58(4), 449-451.

Penn, D.L., Sanna, L.J., y Roberts, D.L., (2008). Social cognition in schizophrenia: an overview. Schizophrenia Bulletin, 34(3), 408-411.

Peralta-Martín, V., y Cuesta-Zorita, M.J. (1994). Validation of positive and negative symptom scale (PANSS) in a sample of Spanish schizophrenic patients. Actas Luso Esp Neurol Psiquiatr Cienc Afines, 22(4), 171-177. Spanish.

Reitan, R.M., y Wolfson, D. (1993). The Halstead-Reitan Neuropsychological Test Battery: Theory and clinical interpretation (2nd ed.). Tucson: Neuropsychology Press.

Revheim, N., Schechter, I., Kim, D., Silipo, G., Allingham, B., Butler, P. y Javitt, D.C. (2006). Neurocognitive and symptom correlates of daily problem-solving skills in schizophrenia. Schizophr Res. 83, 237-245.

Savla, G.N, Vella, L., Armstrong, C.C., Penn., D.L. y Twamle E.W. (2013). Deficits in Domains of Social Cognition in Schizophrenia: A Meta-Analysis of the Empirical Evidence. Schizophrenia Bulletin, 39, 979-992.

Simpson, G.M., y Angus, J.W.S. (1970). A rating scale for extrapyramidal side effects. Acta Psychiatr Scand, 212, 11-19.

Tsotsi, S., Bozikas, V.P., y Kosmidis, M.H. (2015). The role of attention processes in facial affect recognition in schizophrenia. Cogn Neuropsychiatry. 20(6), 526-541.

Recibido: 12 de septiembre 2017 Recepción modificationes: 11 de octubre 2017

Aceptado: 30 de octubre 2017 